Article

\title{
Ion Acceleration by Short Chirped Laser Pulses
}

\author{
Jian-Xing Li ${ }^{1}$, Benjamin Galow ${ }^{2}$, Christoph H. Keitel ${ }^{1}$ and Zoltán Harman ${ }^{1, *}$ \\ ${ }^{1}$ Max Planck Institute for Nuclear Physics, Saupfercheckweg 1, 69029 Heidelberg, Germany; \\ E-Mails: Jian-Xing.Li@mpi-hd.mpg.de (J.-X.L.); Keitel@mpi-hd.mpg.de (C.H.K.) \\ ${ }^{2}$ Ziegelhäuser Landstraße 19, 69120 Heidelberg, Germany; E-Mail: Benjamin.Galow @arcor.de \\ * Author to whom correspondence should be addressed; E-Mail: Zoltan.Harman@ mpi-hd.mpg.de; \\ Tel.: +49-6221-516-170; Fax: +49-6221-516-152.
}

Academic Editor: Ken Ledingham

Received: 18 December 2014 / Accepted: 12 February 2015 / Published: 17 February 2015

\begin{abstract}
Direct laser acceleration of ions by short frequency chirped laser pulses is investigated theoretically. We demonstrate that intense beams of ions with a kinetic energy broadening of about $1 \%$ can be generated. The chirping of the laser pulse allows the particles to gain kinetic energies of hundreds of $\mathrm{MeVs}$, which is required for hadron cancer therapy, from pulses of energies in the order of $100 \mathrm{~J}$. It is shown that few-cycle chirped pulses can accelerate ions more efficiently than long ones, i.e., higher ion kinetic energies are reached with the same amount of total electromagnetic pulse energy.
\end{abstract}

Keywords: laser acceleration; chirped pulses; ion acceleration

PACS classifications: $52.38 . \mathrm{Kd}$, 37.10.Vz, 42.65.-k, 52.75.Di, 52.59.Bi, 52.59.Fn, 41.75.Jv, 87.56.bd

\section{Introduction}

The interaction of intense laser pulses with solids has recently attracted considerable interest. This is largely due to its potential application for accelerating charged particles [1-22]. Tumor therapy with accelerated ion beams (see e.g., [23-28]) would in particular benefit from the replacement of conventional accelerators by all-optical devices, which may become compact and inexpensive in future, allowing this form of cancer therapy to be accessible for more patients. 
In this article, we demonstrate the feasibility of generating ion beams by shining an appropriately chirped short laser pulse on a target. At sufficiently high laser intensities the electrons are quickly ionized, and the ions get directly accelerated by the laser field. Modulating the frequency of the pulse leads to efficient particle energy gain from the field, as it was shown before [29-32]. Here we consider the case of short chirped pulses, when the time duration of the pulse is comparable to a single cycle. We found that acceleration by such short pulses may be more efficient than by long chirped pulses, i.e., the same final ion kinetic energies can be reached with a lower pulse energy.

The acceleration scheme is shown in Figure 1. We assume a solid-density or underdense target consisting of carbon ions, typically, bare nuclei, which can be generated via ionization by an intense pre-pulse. Collective plasma effects become important when the target thickness $L$ in propagation direction of the laser is longer than the wave length of the induced plasma wave, i.e., $\lambda_{p}=2 \pi c / \omega_{p}$ with plasma frequency $\omega_{p}^{2}=4 \pi n_{e} e^{2} / m_{e}$. In the last formula, $n_{e}$ is the electron density, $e$ is the electron charge and $m_{e}$ is the mass of an electron. When interacting with the accelerating pulse, first the lighter electrons are accelerated; they are pushed in the forward direction, as shown previously in [29]. The electrons are followed by the carbon ions, which are directly accelerated by the chirped pulse.
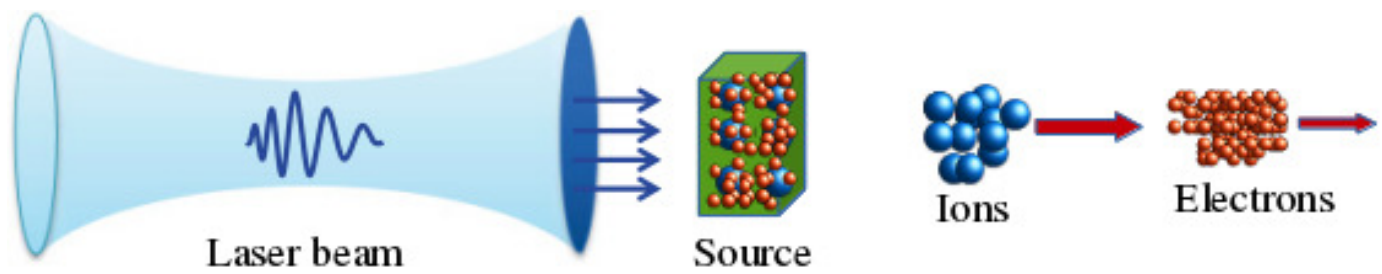

Figure 1. A schematic view of the studied laser acceleration setup. A chirped few-cycle laser pulse is shot on a target. The ionized electrons are accelerated fast in the forward direction, which is followed by the more inert ions, also directly accelerated by the laser pulse. Note that the transverse target size is magnified for illustration purposes. In the simulations it is much smaller than the focal radius of the laser beam.

\section{Laser Acceleration Simulations}

In order to assess the efficiency of laser acceleration by means of few-cycle chirped pulses, we perform simulations based on the classical relativistic equations of motion. In the following subsection, we provide a description of short laser pulses, based on earlier works [33,34]. Subsequently, we describe the particle dynamics in the presence of such intense chirped pulses.

\subsection{Description of Few-Cycle Chirped Focused Laser Pulses}

We consider a circularly polarized laser field propagating along the $z$-direction. Like in [35], the vector potential for the focused ultra-short laser beam is represented as $\boldsymbol{A}=\frac{E_{0}}{k_{0}}\left(\hat{x} \psi(\mathbf{r}, \eta)+i \hat{y} \psi(\mathbf{r}, \eta) e^{i \pi / 2}\right) e^{i \eta}$. Here, the following notations have been introduced: the electric field amplitude is $E_{0}=4 \sqrt{P / c} / w_{0}$, where $P$ is the peak power of a laser pulse, $c$ stands for the light velocity in vacuum, and $w_{0}$ is the laser focal radius. The phase of the field is given as $\eta=\omega_{0} t-k_{0} z$. The wave number is $k_{0}=\omega_{0} / c$, where $\omega_{0}$ is the original (unchirped) frequency of laser at the focal 
point. When the chirping effect is taken into account, the laser frequency and radius have to be modified as follows: $\omega=\omega_{0}(1+b \eta)$, and $w=w_{0} /(1+b \eta)$, where $b$ is the dimensionless chirp parameter. In these formulas, we introduced the quantities $\psi=f\left(1+i \eta / s^{2}\right) e^{i \phi_{0}-f \rho^{2}-\eta^{2} /\left(2 s^{2}\right)}, f=i /\left(i+\nu / z_{r}\right)$, $\nu=z+\eta /(2 k), \rho=r / w, r=\sqrt{x^{2}+y^{2}}, s=\omega_{0} \tau / 2 \sqrt{2 \log 2}$, and $\tau$ is pulse duration. Furthermore, $z_{r}=k w^{2} / 2$ is the Rayleigh length, $k=\omega / c$, and $\phi_{0}$ is a constant phase. When introducing the frequency modulation, the phase parameter $\eta$ and the pulse length parameter $s$ do not need to be modified. Here, $\hat{x}$ and $\hat{y}$ stand for unit vectors in the $x$ and $y$ directions orthogonal to the lasers propagation direction.

Note that the temporal envelope of the laser beam is not factorized in this pulsed solution of the wave equation. The scalar potential $\phi$ is thought to have a similar expression as that of the vector potential $\boldsymbol{A}$, and it can be calculated from the Lorentz gauge condition $\partial \phi / \partial t+\nabla \cdot \boldsymbol{A}=0$. The electromagnetic fields are derived from $\boldsymbol{E}=-\partial \boldsymbol{A} / \partial t-\nabla \phi, \boldsymbol{B}=\nabla \times \boldsymbol{A}: \boldsymbol{E}=\boldsymbol{E}^{(\hat{x})}+\boldsymbol{E}^{(\hat{y})}, \boldsymbol{B}=\boldsymbol{B}^{(\hat{x})}+\boldsymbol{B}^{(\hat{y})}$, herewith,

$$
\begin{aligned}
& E_{x}^{(\hat{x})}=\frac{-E_{1}}{C_{1}^{2}}\left[C_{1}^{3}-f^{2} C_{1} \frac{x^{2}}{z_{r}^{2}}+\frac{f C_{1}}{k z_{r}}-\frac{2 i f k x^{2}}{z_{r}\left(2 k C_{2}+\eta\right)^{2}}\right] \\
& E_{y}^{(\hat{x})}=\frac{E_{1} f x y}{C_{1}^{2} z_{r}^{2}}\left[f C_{1}+\frac{2 i k z_{r}}{\left(2 k j_{z}+\eta\right)^{2}}\right] \\
& E_{z}^{(\hat{x})}=\frac{-E_{1} f x}{C_{1}^{2} z_{r}^{2}}\left[\frac{i f C_{1}}{k}-C_{1} z_{r}\left(i+\frac{i f^{2} r^{2}}{4 z_{r}^{2}}-\frac{\eta}{s^{2}}\right)-\frac{i C_{1} z_{r}}{s^{2}+i \eta}-z_{r} C_{2}\right] \\
& B_{x}^{(\hat{x})}=0 \\
& B_{y}^{(\hat{x})}=E_{1}\left(\frac{i f}{2 k z r}-i-\frac{i f^{2} r^{2}}{4 z_{r}^{2}}+\frac{\eta}{s^{2}}+\frac{1}{i s^{2}-\eta}\right) \\
& B_{z}^{(\hat{x})}=\frac{E_{1} f y}{z_{r}}
\end{aligned}
$$

and, furthermore,

$$
\begin{aligned}
& E_{y}^{(\hat{y})}=\frac{-E_{1}}{C_{1}^{2}}\left[C_{1}^{3}-f^{2} C_{1} \frac{y^{2}}{z_{r}^{2}}+\frac{f C_{1}}{k z_{r}}-\frac{2 i f k y^{2}}{z_{r}\left(2 k C_{2}+\eta\right)^{2}}\right] \\
& E_{x}^{(\hat{y})}=\frac{E_{1} f x y}{C_{1}^{2} z_{r}^{2}}\left[f C_{1}+\frac{2 i k z_{r}}{\left(2 k j_{z}+\eta\right)^{2}}\right] \\
& E_{z}^{(\hat{y})}=\frac{-E_{1} f y}{C_{1}^{2} z_{r}^{2}}\left[\frac{i f C_{1}}{k}-C_{1} z_{r}\left(i+\frac{i f^{2} r^{2}}{4 z_{r}^{2}}-\frac{\eta}{s^{2}}\right)-\frac{i C_{1} z_{r}}{s^{2}+i \eta}-z_{r} C_{2}\right] \\
& B_{y}^{(\hat{y})}=0 \\
& B_{x}^{(\hat{y})}=-E_{1}\left(\frac{i f}{2 k z r}-i-\frac{i f^{2} r^{2}}{4 z_{r}^{2}}+\frac{\eta}{s^{2}}+\frac{1}{i s^{2}-\eta}\right) \\
& B_{z}^{(\hat{y})}=\frac{-E_{1} f x}{z_{r}}
\end{aligned}
$$

where $E_{1}=E_{0} \psi e^{i\left(\eta+b \eta^{2}\right)} / S_{0}$, with the normalization parameter $S_{0}=\left[-s_{1}^{2}-1 /\left(k z_{r}\right)\right] / s_{1}$, $s_{1}=i\left[1+1 /\left(2 k z_{r}\right)+1 / s^{2}\right], j_{z}=z+i z_{r}$, and the following shorthand notations have been introduced:

$$
\begin{aligned}
C_{1} & =i+\frac{i k^{2} r^{2}-2 k j_{z}-\eta}{\left(2 k j_{z}+\eta\right)^{2}}+\frac{s^{2}+i \eta s^{2}-\eta^{2}}{s^{2}\left(\eta-i s^{2}\right)} \\
C_{2} & =\frac{-1}{\left(2 k j_{z}+\eta\right)^{2}}+\frac{4 k j_{z}+2 \eta-2 i k^{2} r^{2}}{\left(2 k j_{z}+\eta\right)^{3}}+\frac{\eta^{2}-s^{2}-i \eta s^{2}}{\left(i \eta s+s^{3}\right)^{2}}+\frac{2 i \eta+s^{2}}{i \eta s^{2}+s^{4}}
\end{aligned}
$$


These field expressions are in concordance with the sub-cycle pulse field of [36] and the long-pulse field of [37]. Such fields were also employed recently to simulate quantum radiation reaction effects for an ensemble of electrons interacting with ultra-short pulses [34]. For the much slower ions studied here, the influence of radiation reaction can be neglected [15].

\subsection{Particle Dynamics}

The time-dependent dynamics of an ensemble of interacting ions is considered. An ion indexed by $j$, of mass $m$ and charge $q$ is accelerated to relativistic energy and momentum, respectively, of $\mathcal{E}_{j}=\gamma_{j} m c^{2}$ and $\boldsymbol{p}_{j}=\gamma_{j} m c \boldsymbol{\beta}_{j}$, where $\boldsymbol{\beta}_{j}$ is the velocity of the particle divided by $c$, the velocity of light in vacuum, and $\gamma=\left(1-\beta^{2}\right)^{-1 / 2}$ is the Lorentz factor, when interacting with the time-dependent fields $\boldsymbol{E}$ and $\boldsymbol{B}$ of a laser pulse. Thus, the dynamics is described by the coupled Newton-Lorentz equations, given in SI (International System of Units):

$$
\begin{aligned}
\frac{d \boldsymbol{p}_{j}}{d t} & =q\left(\boldsymbol{E}\left(\boldsymbol{r}_{j}\right)+\boldsymbol{E}_{j}^{\text {int. }}+c \boldsymbol{\beta}_{j} \times\left(\boldsymbol{B}\left(\boldsymbol{r}_{j}\right)\right)\right) \\
\frac{d \mathcal{E}_{j}}{d t} & =q c \boldsymbol{\beta}_{j} \cdot\left(\boldsymbol{E}\left(\boldsymbol{r}_{j}\right)+\boldsymbol{E}_{j}^{\text {int. }}\right)
\end{aligned}
$$

The electric field of the inter-ionic interaction is approximated by $\boldsymbol{E}_{j}^{\mathrm{int}}=-\sum_{k \neq j} \nabla \phi_{j k}$, with the Coulomb interaction scalar potential

$$
\phi_{j k}=\frac{q}{4 \pi \epsilon_{0}} \frac{1}{\left|\boldsymbol{r}_{j}-\boldsymbol{r}_{k}\right|}
$$

Here, $\epsilon_{0}$ is the vacuum permittivity. The presence of plasma electrons can be neglected when simulating the ions' acceleration dynamics, as they are blown off first by the pulse, as it was shown by particle-in-cell simulations [29].

Since the electromagnetic fields have a complex mathematical structure, and the inclusion of the ions' interaction leads to coupled motion, one has to solve the above differential equations numerically. A numerical integration of Equation (4) yields the particles' velocities $\boldsymbol{\beta}_{j}$ and thus also their energy gain at a final time equal to many laser cycles.

\section{Results and Discussion}

We first present the calculation results for a single test ion. Bare carbon nuclei $\left(\mathrm{C}^{6+}\right.$ ions $)$ are chosen here because of their higher contrast of dose deposition in the tissue as compared to protons. In order to find the optimal parameters of the pulse and the optimal initial velocity of the ion, we performed numerical simulations by calculating the energy gain for a particle as a function of the chirp parameter $b$ and the initial energy $E_{0}=\gamma_{0} m c^{2}$ of the ion. The results are presented in Figure 2. The optimal chirping is in the range around $b= \pm 0.1$, with low initial kinetic energies around $\gamma_{0} \approx 1.05$. We note that for the circularly polarized pulses employed here, the dependence of the energy on the chirping parameter is a slowly-varying function. This is not the case for linearly polarized fields, where this function shows a strong oscillatory behavior [29]. Therefore, the appropriate chirping can be more practically implemented in experiments. The maximal energy gain that can be reached in this setting is around $100 \mathrm{MeV} / \mathrm{u}$, reaching the range of interest in medical applications. 


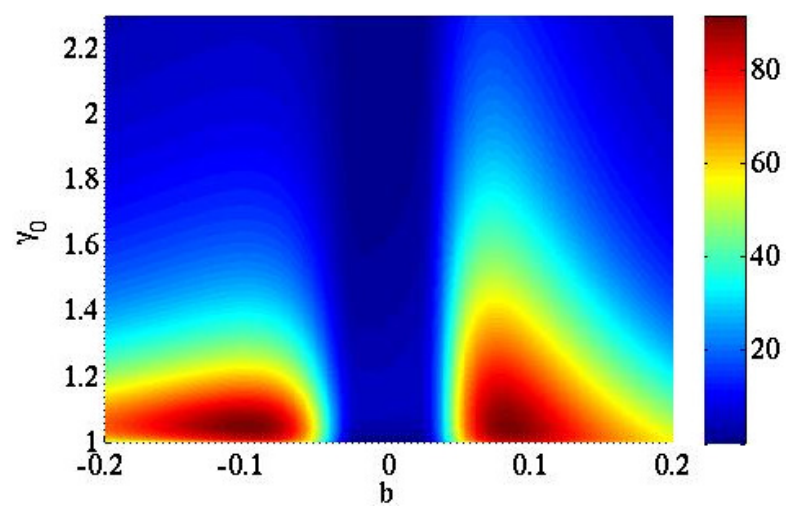

Figure 2. Energy gain in units of $\mathrm{MeV} / \mathrm{u}$ with dependence on the chirp parameter $b$ and the initial dimensionless energy parameter $\gamma_{0}$ (i.e., Lorentz factor, $E_{0}=\gamma_{0} m c^{2}$ ). The wavelength of the laser is chosen to be $1 \mu \mathrm{m}$ and its power to $10 \mathrm{PW}$. The pulse duration is equal to 3 laser cycles, and the focal waist radius is equal to $\lambda$. A circularly polarized laser pulse is employed.

As the previous figure shows, the acceleration by a chirped short pulse is optimal when the initial kinetic energy of particles is low. Motivated by this, we separately consider the experimentally advantageous situation when the particles are initially at rest, i.e., $\gamma_{0}=1$. The energy gain is shown in Figure 3 with dependence of the chirp parameter $b$. The maximal gain that can be achieved by such pulses when the particles are at rest initially is approximately $80 \mathrm{MeV} / \mathrm{u}$. In the case of circularly polarized fields, similar gains can be reached when applying a negative frequency chirp $(b<0)$, i.e., when the carrier frequency of the laser decreases in time. Additionally, circularly polarized laser beams have the advantage over linearly polarized beams that they are not sensitive to small variations of the chirp parameter $b-c f$. Figure 3 with Figure 2 of Ref. [29]. Figure 4 shows, for an optimal chirp parameter $b=0.089$, the energy gain and the corresponding pulse as a function of the longitudinal displacement $z$, confirming that the final kinetic energy around $100 \mathrm{MeV}$ is reached on a sub-wavelength scale. The figure also illustrates — in accordance with earlier findings [29] — that it is the asymmetric part of the pulse, induced by the strong frequency modulation, that causes sudden acceleration of the ion. This is in contrast to an unchirped laser pulse, where a charged particle would gain kinetic energy during the first half of a laser cycle and subsequently loses this energy during the second half of the respective cycle due to the symmetry of the pulse. However, we want to emphasize that frequency modulation over a large frequency bandwidth is presently experimentally accessible only at lower field intensities [38,39].

In order to find the optimal pulse duration for acceleration, we performed single-particle simulations calculating the energy gain of a particle initially at rest, with dependence on this parameter, at a fixed total pulse energy. The results are displayed in Figure 5a, showing that maximal efficiency can be reached for pulses with a time duration of 3 to 4 cycles. Increasing the pulse duration can only decrease the final energy gain, since the peak electric field of the pulse decreases if the energy of the pulse is distributed over a longer time. Furthermore, in pulses with durations below 3-4 periods, the ion does not spend sufficient time interacting with the field to reach its maximal velocity. For each pulse duration, the chirp parameter $b$ was optimized independently. The corresponding values of the chirp parameters are displayed in Figure $5 b$. 


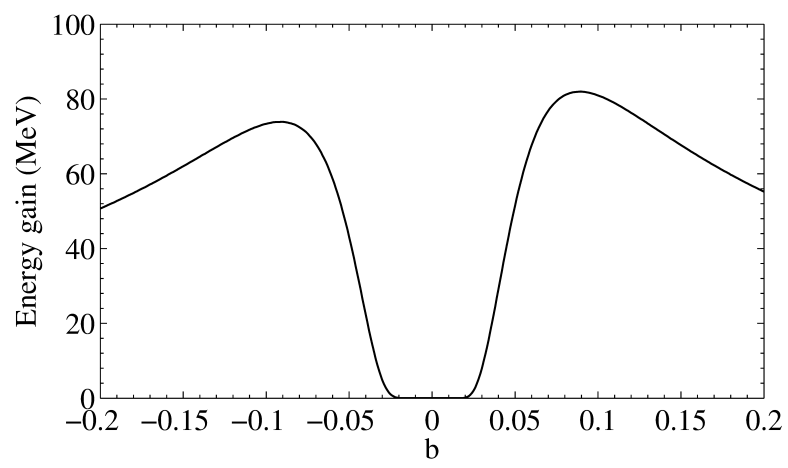

Figure 3. Energy gain per nucleon for $\mathrm{C}^{6+}$ nuclei as a function of the chirp parameter $b$. The nuclei are assumed to be initially at rest, i.e., $\gamma_{0}=1$. Other parameters are as described for Figure 2.

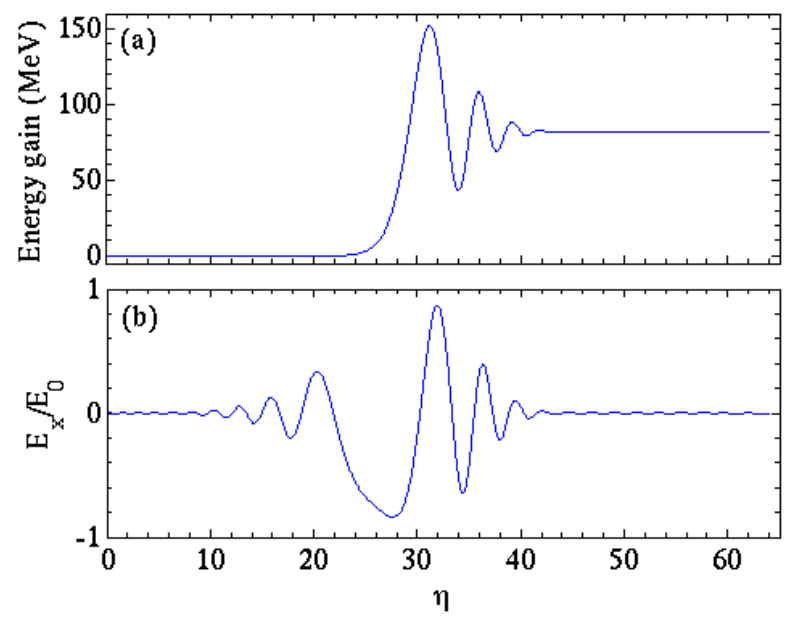

Figure 4. (a) Energy gain per nucleon for $\mathrm{C}^{6+}$ nuclei and (b) the electric field of the pulse as a function of the phase $\eta$, for a fixed optimal chirp parameter of $b=0.089$. Other parameters are as described for Figure 2.

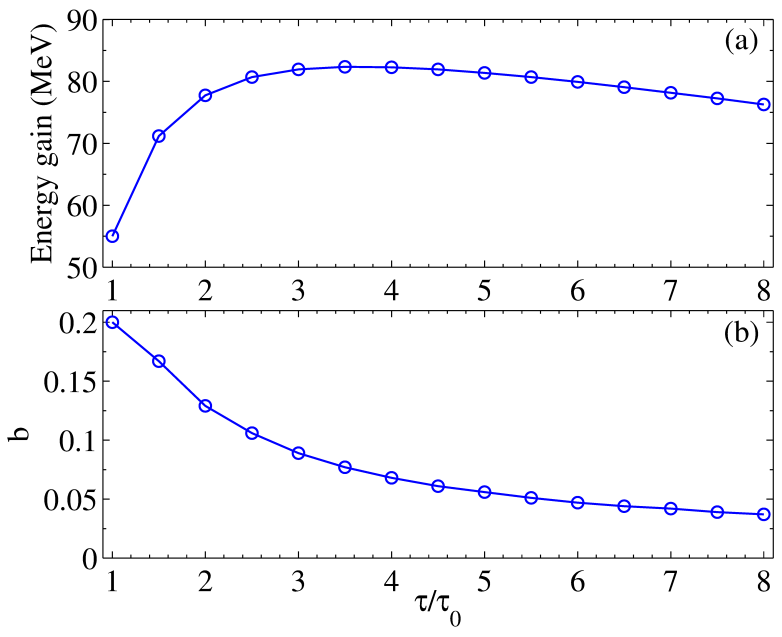

Figure 5. (a) Energy gain per nucleon for $\mathrm{C}^{6+}$ nuclei and (b) optimal chirp parameter $b$ as a function of the pulse duration $\tau$, given in units of the period $\tau_{0}$. In these calculations, the pulse energy was kept fixed at a value of $100 \mathrm{~J}$. 
The energy distribution of a beam of ions generated by laser acceleration was determined by many-particle calculations based on the coupled Newton-Lorentz equations (Equation (4)). We assume that the ions are randomly distributed in a small nano-scale cylindrical volume before interacting with the short focused laser pulse. The ions were assumed to be initially at rest, and have a solid-state density $\left(2.4 \times 10^{24}\right.$ ions $\left./ \mathrm{cm}^{3}\right)$. Simulations were performed with and without the inclusion of the ion-ion interaction to assess the effect of Coulomb repulsion on the energy gain and its broadening. In both cases, the set of random initial coordinates utilized was kept constant. Results are shown in Figure 6. For this case, collective plasma effects are negligible since the induced plasma wavelength, $\lambda_{p}=0.0088 \mu \mathrm{m}$, is much larger than the target thickness, $L=0.0004 \mu \mathrm{m}$. Including the particle-particle repulsion yields an order-of-magnitude broader distribution than the simulation with artificially switching off the interaction. However, the average kinetic energy is not influenced by this: in the first, realistic simulation, the average gain is $82.557 \mathrm{MeV} / \mathrm{u}$, with a standard deviation of $1.042 \mathrm{MeV} / \mathrm{u}(1.262 \%)$; in the latter case, the average gain is the same, while the standard deviation is $0.006 \mathrm{MeV} / \mathrm{u}(0.007 \%)$. Thus, we may conclude that for dense targets, it is indeed necessary to include inter-particle interactions in realistic simulations, even at the very high laser intensities considered. The beam energy spread is in the medically applicable range, i.e., approximately $1 \%$, enabled by the small size of the target. The trajectories of the overdense interacting $\mathrm{C}^{6+}$ ion beam are illustrated in Figure 7, and the emission angle is approximately 0.05 (radian), i.e., 2.87 (degree). Please note that for the angle we use the unit of radian in what follows. For the same conditions, except taking a larger chirp parameter, $b=0.1$, the emission angle slightly increases from 0.05 to 0.051 . Besides, for a longer laser pulse with the pulse duration $\tau=4 \tau_{0}$, the emission angle decreases to 0.048 .
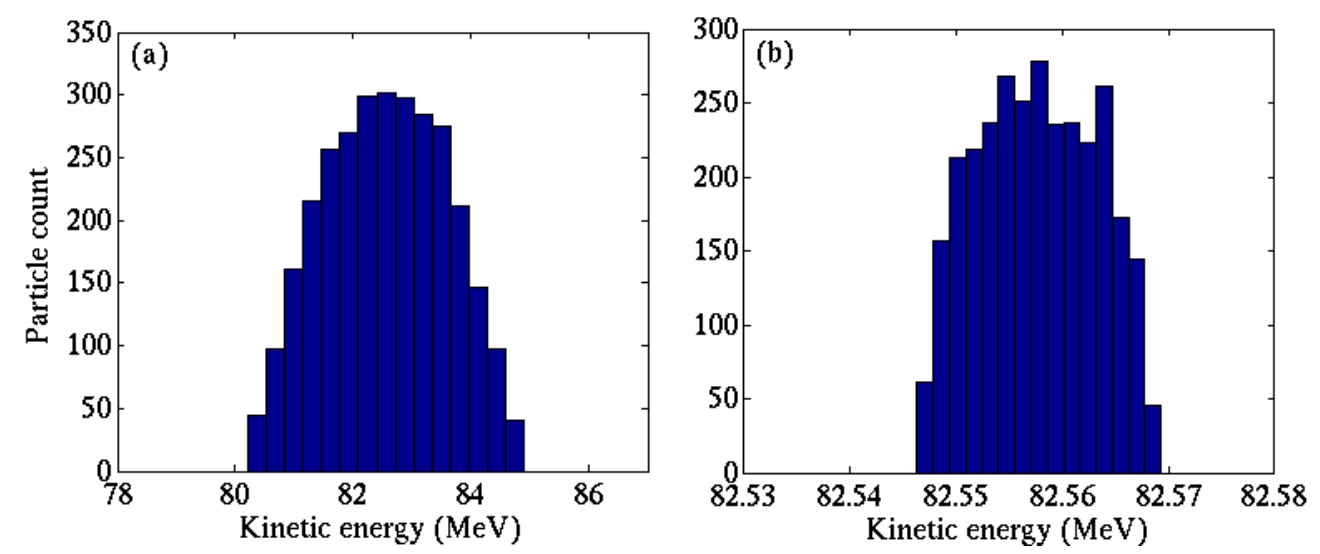

Figure 6. (a) Energy distribution histogram for an ensemble of 3000 interacting $\mathrm{C}^{6+}$ ions, confined initially in a small cylinder (length $0.0004 \mu \mathrm{m}$, diameter $0.002 \mu \mathrm{m}$ ); (b) Energy distribution for the same set of ions, with the inter-particle Coulomb repulsion switched off. Note the different energy scales of the two sub-figures.

In addition to a nano-scale target, simulations were made for a more extended underdense carbon plasma target, which may be realized as an expanding plasma created by a pre-pulse. The kinetic energy distribution for a target of ions with an initial density of $2.4 \times 10^{20}$ ions $/ \mathrm{cm}^{3}$ is presented as a histogram in Figure 8. The induced plasma wavelength is $\lambda_{p}=0.88 \mu \mathrm{m}$ and the target thickness is $L=0.04 \mu \mathrm{m}$. Therefore, collective plasma effects are ignored. In this case, particle-particle 
interactions are weaker, and thus the results are less sensitive to the presence of interaction. We obtain an average ion gain of $82.553 \mathrm{MeV} / \mathrm{u}$, with a standard deviation of $0.138 \mathrm{MeV} / \mathrm{u}(0.167 \%)$ for the more realistic simulation with the ions' mutual repulsion taken into account, and to the same average gain with a spread of $0.069 \mathrm{MeV} / \mathrm{u}(0.083 \%)$ with the interaction neglected. The energies reached here coincide with the results for a solid-density ion plasma target, but the energy broadening is somewhat better. The trajectories of the underdense interacting $\mathrm{C}^{6+}$ ion beam are illustrated in Figure 9. The emission angle is approximately 0.005 , i.e., 10 times smaller than that of the overdense case.

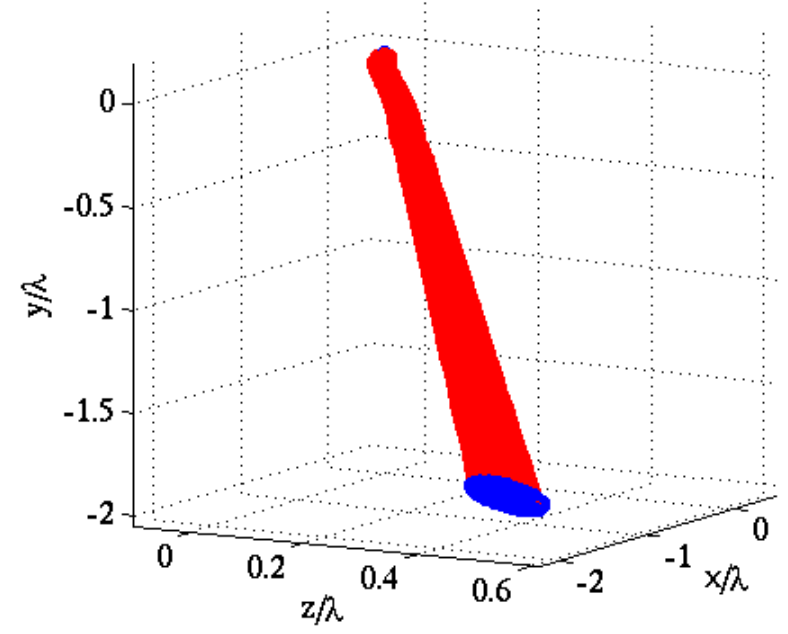

Figure 7. Trajectories of the overdense interacting $\mathrm{C}^{6+}$ ion beam. The red curves are the trajectories of $\mathrm{C}^{6+}$ ions, and blue points are the corresponding initial and final positions in the simulations with $-4 s \leq \eta \leq 4 s$. The parameters employed here are the same as those of Figure 6a.
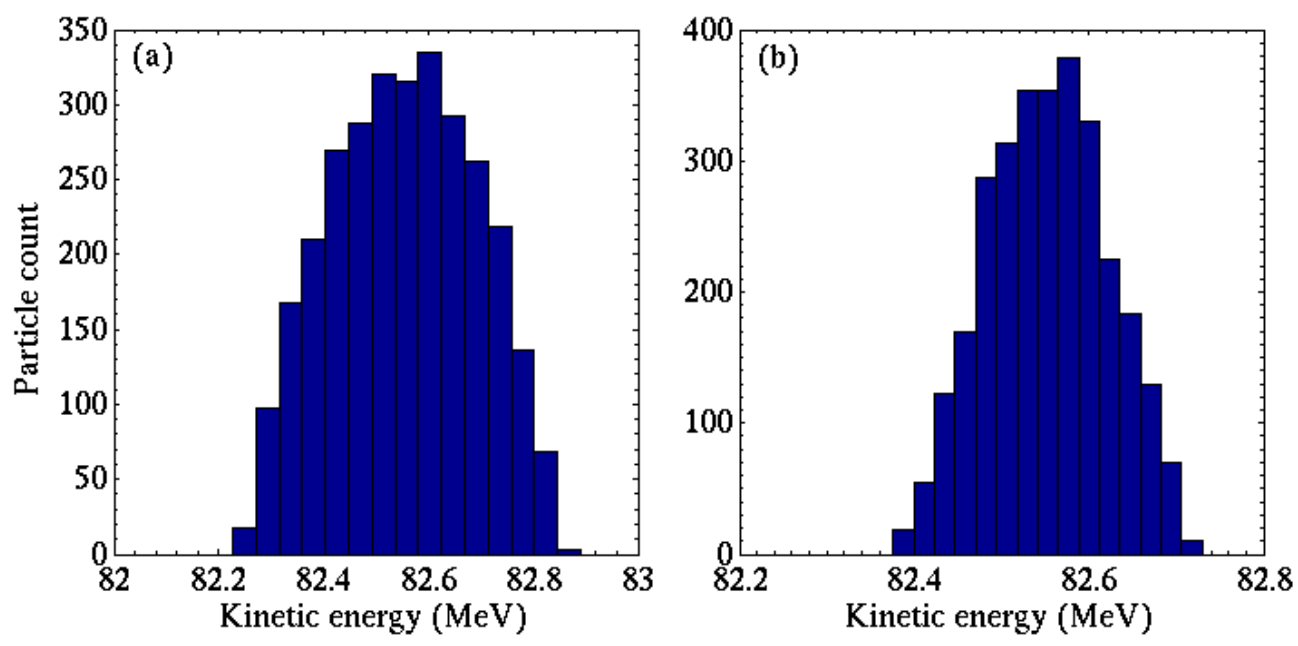

Figure 8. (a) Energy distribution histogram for the case of an underdense target formed by 3000 interacting $\mathrm{C}^{6+}$ ions, confined initially in a cylinder (length $0.04 \mu \mathrm{m}$, diameter $0.02 \mu \mathrm{m}$ ); (b) Energy distribution for the same underdense target, with the inter-particle interaction neglected. 


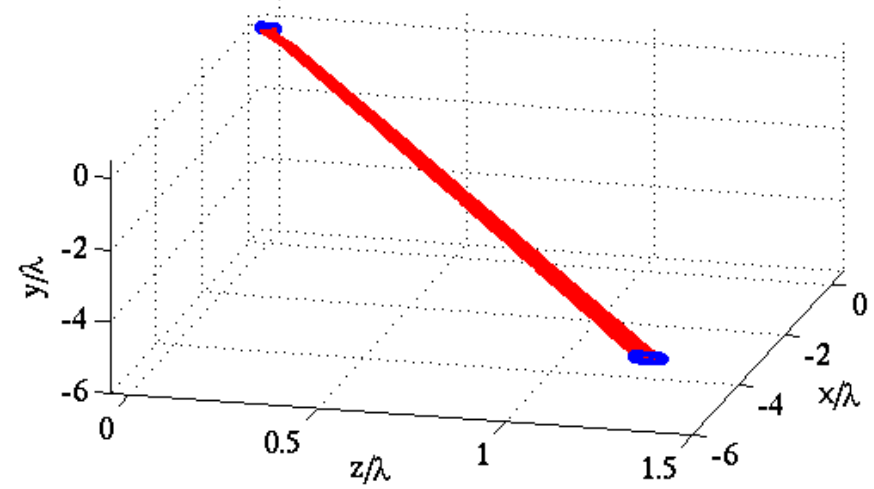

Figure 9. Trajectories of the underdense interacting $\mathrm{C}^{6+}$ ion beam. The red curves are the trajectories of $\mathrm{C}^{6+}$ ions, and blue points are the corresponding initial and final positions in the simulations with $-4 s \leq \eta \leq 12 s$. The parameters employed here are the same as those of Figure 8a.

\section{Conclusions}

Relativistic many-particle simulations were performed in order to assess the applicability of few-cycle chirped laser pulses to ion acceleration for the purpose of hadron cancer therapy. An accurate description of the temporal and spatial structure of the laser fields was employed. We have found that such pulses with durations of 3-4 cycles, when focused on small underdense or solid-density plasma targets, can produce ion beams with properties in the range of medical requirements. They are also more efficient in accelerating ions than longer chirped pulses investigated in our earlier work [29]: a further increase of pulse duration beyond the optimal value decreases the particles' energy gain, as the pulse energy distributed over longer times leads to weaker accelerating fields.

\section{Acknowledgments}

The authors acknowledge insightful conversations with Yousef I. Salamin. The first author J.-X.L. is partially supported by the National Natural Science Foundation of China (Grant No. 11304077).

\section{Author Contributions}

J.-X.L. conceived the underlying idea of the paper. J.-X.L. and B.G. performed the numerical calculations. Z.H. and J.-X.L. wrote the paper. All authors analyzed and discussed the simulation results.

\section{Conflicts of Interest}

The authors declare no conflict of interest. 


\section{References}

1. Schwoerer, H.; Pfotenhauer, S.; Jäckel, O.; Amthor, K.U.; Liesfeld, B.; Ziegler, W.; Sauerbrey, R.; Ledingham, K.W.D.; Esirkepov, T. Laser-plasma acceleration of quasi-monoenergetic protons from microstructured targets. Nature 2006, 439, 445-448.

2. Hegelich, B.M.; Albright, B.J.; Cobble, J.; Flippo, K.; Letzring, S.; Paffett, M.; Ruhl, H.; Schreiber, J.; Schulze, R.K.; Fernández, J.C. Laser acceleration of quasi-monoenergetic MeV ion beams. Nature 2006, 439, 441-444.

3. Fuchs, J.; Antici, P.; d'Humières, E.; Lefebvre, E.; Borghesi, M.; Brambrink, E.; Cecchetti, C.A.; Kaluza, M.; Malka, V.; Manclossi, M.; et al. Laser-driven proton scaling laws and new paths towards energy increase. Nat. Phys. 2006, 2, 48-54.

4. Esirkepov, T.; Yamagiwa, M.; Tajima, T. Laser ion-acceleration scaling laws seen in multiparametric particle-in-cell simulations. Phys. Rev. Lett. 2006, 96, 105001.

5. Robson, L.; Simpson, P.T.; Clarke, R.J.; Ledingham, K.W.D.; Lindau, F.; Lundh, O.; McCanny, T.; Mora, P.; Neely, D.; Wahlström, C.G.; et al. Scaling of proton acceleration driven by petawatt-laser-plasma interactions. Nat. Phys. 2007, 3, 58-62.

6. Maksimchuk, A.; Gu, S.; Flippo, K.; Umstadter, D.; Bychenkov, V.Y. Forward ion ccceleration in thin films driven by a high-intensity laser. Phys. Rev. Lett. 2000, 84, 4108-4111.

7. Snavely, R.A.; Key, M.H.; Hatchett, S.P.; Cowan, T.E.; Roth, M.; Phillips, T.W.; Stoyer, M.A.; Henry, E.A.; Sangster, T.C.; Singh, M.S.; et al. Intense high-energy proton beams from petawatt-laser irradiation of solids. Phys. Rev. Lett. 2000, 85, 2945-2948.

8. Karsch, S.; Düsterer, S.; Schwoerer, H.; Ewald, F.; Habs, D.; Hegelich, M.; Pretzler, G.; Pukhov, A.; Witte, K.; Sauerbrey, R. High-intensity laser induced ion acceleration from heavy-water droplets. Phys. Rev. Lett. 2003, 91, 015001.

9. Romagnani, L.; Fuchs, J.; Borghesi, M.; Antici, P.; Audebert, P.; Ceccherini, F.; Cowan, T.; Grismayer, T.; Kar, S.; Macchi, A.; et al. Dynamics of electric fields driving the laser acceleration of multi-MeV protons. Phys. Rev. Lett. 2005, 95, 195001.

10. Cowan, T.E.; Fuchs, J.; Ruhl, H.; Sentokua, Y.; Kempa, A.; Audebertb, P.; Roth, M.; Stephens, R.; Barton, I.; Blazevic, A.; et al. Ultra-low emittance, high current proton beams produced with a laser-virtual cathode sheath accelerator. Nuclear Instrum. Methods Phys. Res. 2005, 544, 277-284.

11. Albright, B.J.; Yin, L.; Hegelich, B.M.; Bowers, K.J.; Kwan, T.J.T.; Fernández, J.C. Theory of laser acceleration of light-ion beams from interaction of ultrahigh-intensity lasers with layered targets. Phys. Rev. Lett. 2006, 97, 115002.

12. Tajima, T.; Dawson, J.M. Laser electron accelerator. Phys. Rev. Lett. 1979, 43, 267-270.

13. Badziak, J. Laser-driven generation of fast particles. Opto-Electron. Rev. 2007, 15, 1-12.

14. Salamin, Y.I.; Harman, Z.; Keitel, C.H. Direct high-power laser acceleration of ions for medical applications. Phys. Rev. Lett. 2008, 100, 155004.

15. Galow, B.J.; Harman, Z.; Keitel, C.H. Intense high-quality medical proton beams via laser fields. Opt. Express 2010, 18, 25950-25957.

16. Salamin, Y.I.; Hu, S.X.; Hatsagortsyan, K.Z.; Keitel, C.H. Relativistic high-power laser-matter interactions. Phys. Rep. 2006, 427, 41-155. 
17. Di Piazza, A.; Müller, C.; Hatsagortsyan, K.Z.; Keitel, C.H. Extremely high-intensity laser interactions with fundamental quantum systems. Rev. Mod. Phys. 2012, 84, 1177-1228.

18. Peralta, E.A.; Soong, K.; England, R.J.; Colby, E.R.; Wu, Z.; Montazeri, B.; McGuinness, C.; McNeur, J.; Leedle, K.J.; Walz, D.; et al. Demonstration of electron acceleration in a laser-driven dielectric microstructure. Nature 2013, 503, 91-94.

19. Haberberger, D.; Tochitsky, S.; Fiuza, F.; Gong, C.; Fonseca, R. Collisionless shocks in laser-produced plasma generate monoenergetic high-energy proton beams. Nat. Phys. 2012, 8, 95-99.

20. Hooker, S.M. Developments in laser-driven plasma accelerators. Nat. Photonics 2013, 7, 775-782.

21. Zigler, A.; Eisenman, S.; Botton, M.; Nahum, E.; Schleifer, E.; Baspaly, A.; Pomerantz, I.; Abicht, F.; Branzel, J.; Priebe, G.; et al. Enhanced proton acceleration by an ultrashort laser interaction with structured dynamic plasma targets. Phys. Rev. Lett. 2013, 110, 215004.

22. Harman, Z.; Salamin, Y.I.; Galow, B.J.; Keitel, C.H. Optimizing direct intense-field laser acceleration of ions. Phys. Rev. A 2011, 84, 053814.

23. Combs, S.E.; Nikoghosyan, A.; Jäkel, O.; Karger, C.P.; Haberer, T.; Münter, M.W.; Huber, P.E.; Debus, J.; Schulz-Ertner, D. Carbon ion radiotherapy for pediatric patients and young adults treated for tumors of the skull base. Cancer 2009, 115, 1348-1355.

24. Jäkel, O.; Krämer, M.; Karger, C.P.; Debus, J. Treatment planning for heavy ion radiotherapy: Clinical implementation and application. Phys. Med. Biol. 2001, 46, 1101-1116.

25. Yock, T.I.; Tarbell, N.J. Technology Insight: Proton beam radiotherapy for treatment in pediatric brain tumors. Nat. Clin. Pract. Oncol. 2004, 1, 97-103.

26. Levin, M.P.; Kooy, H.; Loeffler, J.S.; DeLaney, T.F. Proton beam therapy. Br. J. Cancer 2005, 93, 849-854.

27. Coen, J.J.; Zietman, A.L. Proton radiation for localized prostate cancer. Nat. Rev. Urol. 2009, 6, 324-330.

28. Fokas, E.; Kraft, G.; An, H.; Engenhart-Cabillic, R. Ion beam radiobiology and cancer: Time to update ourselves. Biochim. Biophy. Acta (BBA) 2009, 1796, 216-229.

29. Galow, B.; Salamin, Y.I.; Liseykina, T.; Harman, Z.; Keitel, C.H. Dense monoenergetic proton beams from chirped laser-plasma interaction. Phys. Rev. Lett. 2011, 107, 185002.

30. Singh, K.P. Electron acceleration by a chirped short intense laser pulse in vacuum. Appl. Phys. Lett. 2005, 87, 254102.

31. Sohbatzadeh, F.; Mirzanejhad, S.; Ghasemi, M. Electron acceleration by a chirped Gaussian pulse. Phys. Plasmas 2006, 13, 123108.

32. Sohbatzadeh, F.; Mirzanejhad, S.; Aku, H. Synchronization scheme in electron vacuum acceleration by a chirped Gaussian pulse. Phys. Plasmas 2009, 16, 023106.

33. Li, J.X.; Salamin, Y.I.; Hatsagortsyan, K.Z.; Keitel, C.H. Fields of an ultrashort tightly-focused laser pulse. Opt. Lett. 2014, submitted.

34. Li, J.X.; Hatsagortsyan, K.Z.; Keitel, C.H. Robust signatures of quantum radiation reaction in focused ultrashort laser pulses. Phys. Rev. Lett. 2014, 113, 044801.

35. Esarey, E.; Sprangle, P.; Pilloff, M.; Krall, J. Theory and group velocity of ultrashort, tightly focused laser pulses. J. Opt. Soc. Am. B 1995, 12, 1695-1703. 
36. Lin, Q.; Zheng, J.; Becker, W. Subcycle pulsed focused vector beams. Phys. Rev. Lett. 2006, 97, 253902.

37. Salamin, Y.I.; Keitel, C.H. Electron acceleration by a tightly focused laser beam. Phys. Rev. Lett. 2002, 88, 095005.

38. Goulielmakis, E.; Koehler, S.; Reiter, B.; Schultze, M.; Verhoef, A.J.; Serebryannikov, E.E.; Zheltikov, A.M.; Krausz, F. Ultrabroadband, coherent light source based on self-channeling of few-cycle pulses in helium. Opt. Lett. 2008, 33, 1407-1409.

39. Wirth, A.; Hassan, M.T.; Grguraš, I.; Gagnon, J.; Moulet, A.; Luu, T.T.; Pabst, S.; Santra, R.; Alahmed, Z.A.; Azzeer, A.M.; et al. Synthesized light transients. Science 2011, 334, 195-200.

(c) 2015 by the authors; licensee MDPI, Basel, Switzerland. This article is an open access article distributed under the terms and conditions of the Creative Commons Attribution license (http://creativecommons.org/licenses/by/4.0/). 